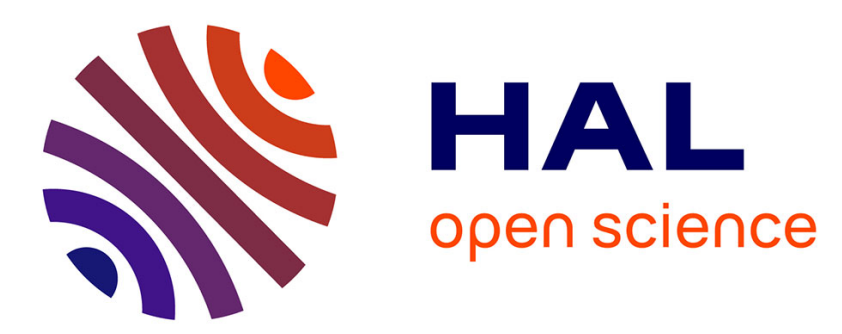

\title{
The role of uniaxial magnetic anisotropy distribution on domain wall tilting in amorphous glass-coated microwires
}

Kornel Richter, Andre Thiaville, Rastislav Varga, Jeffrey Mccord

\section{- To cite this version:}

Kornel Richter, Andre Thiaville, Rastislav Varga, Jeffrey Mccord. The role of uniaxial magnetic anisotropy distribution on domain wall tilting in amorphous glass-coated microwires. Journal of Applied Physics, 2020, 127 (19), pp.193905. 10.1063/5.0005532 . hal-03121207

\section{HAL Id: hal-03121207 \\ https://cnrs.hal.science/hal-03121207}

Submitted on 26 Jan 2021

HAL is a multi-disciplinary open access archive for the deposit and dissemination of scientific research documents, whether they are published or not. The documents may come from teaching and research institutions in France or abroad, or from public or private research centers.
L'archive ouverte pluridisciplinaire HAL, est destinée au dépôt et à la diffusion de documents scientifiques de niveau recherche, publiés ou non, émanant des établissements d'enseignement et de recherche français ou étrangers, des laboratoires publics ou privés. 


\title{
The role of uniaxial magnetic anisotropy distribution on domain wall tilting in amorphous glass-coated microwires
}

\author{
Kornel Richter ${ }^{1,2^{*}}$, André Thiaville ${ }^{3}$, Rastislav Varga ${ }^{2}$, and Jeffrey McCord ${ }^{1}$ \\ ${ }^{1}$ Institute for Materials Science, Kiel University, Kaiserstraße 2, 24143 Kiel, Germany \\ 2 Institute of Physics, P.J. Safarik University, Park Angelinum 9, 04154 Kosice, Slovakia \\ ${ }^{3}$ Laboratoire de Physique des Solides, Univ. Paris-Sud, CNRS UMR 8502, 91405 Orsay, \\ France
}

* author to whom correspondence should be addressed: kornel.richter@gmail.com

Magnetic properties of highly magnetostrictive amorphous glass-coated microwires are strongly correlated to the presence of a glass coating that introduces a spatially inhomogeneous stress field distribution. We investigate the influence of mechanical stresses on the inclination of magnetic domain walls in magnetic microwires. Magneto-optical Kerr effect imaging is used to compare the tilted orientation of the domain wall shape in as-cast and annealed microwires. Angular dependencies of magnetization loops measured by alternating gradient field magnetometry reveal that the change of domain wall tilting with annealing is related to the decrease of a magnetic anisotropy with axial orientation. Finally, micromagnetic simulations are used to show that sufficiently high uniaxial magnetic anisotropy gives rise to the presence of the observed charged domain walls with tilted orientation.

Keywords: Magneto-optical Kerr effect, magnetic anisotropy, magnetic cylinders, Kerr imaging

\section{Introduction}

The mechanisms of a magnetic domain wall propagation have been employed in many emerging applications, such as magnetic sensors [1,2], memories [3,4] or energy harvesting devices for internet of things (IoT) [5]. The suitability of the used magnetic nano- and micro-sized elements stems from the low energy consumption for changing the magnetization states that are resistant against loss of information by energy outage. With a small exception [6], most of the dynamic properties of magnetic domain walls in planar patterned nanostructures can be predicted by a simple one-dimensional (1D) model. Recent progress in advanced fabrication techniques allows preparation of complex threedimensional magnetic wires $[7,8]$ that could provide new classes of domain walls with unprecedented richness and applicability of their dynamics.

Amorphous glass-coated microwires [9] with positive magnetostriction and spontaneous bistability features possess very high domain wall velocities that can reach values of up to $5 \mathrm{~km} / \mathrm{s}[10,11]$. Furthermore, the fast domain wall motion can be tailored effectively by a transverse magnetic field [12] 
or by temperature [13]. Such multi-sensitivity of microwires makes them an ideal candidate for advanced sensors [14,15], which are capable of the measurement of several physical quantities at the same time. Despite some attempts to propose a simple mechanical model of domain wall motion [16,17], the origin of the obtained high domain wall velocity is still not clear. It is evident that a detailed knowledge of the internal spin structure in statics is crucial for understanding the dynamic behavior. The shape of domain walls in microwires has been in focus of several theoretical and experimental studies. Analytical calculations of eddy-current damping mechanism [17] predict a conical shape of the domain wall. On the other hand, analysis of a voltage pulse induced in a single-turn pick-up coil showed [18] that the change of the magnetization is not symmetrical around the principal axis of the cylindrical microwire. A novel method for mapping magnetization in thin cylinders [19] confirmed a tilted domain wall structure at the surface. However, the method suffers from the fact that the domain wall cannot be observed statically, and the field of view is limited by the size of laser spot, which makes an unambiguous interpretation difficult.

In this paper, we focus on the domain wall inclination in FeSiB microwires and study the influence of the axial magnetic anisotropy on the domain wall tilting via magneto-optical Kerr effect (MOKE) microscopy. The manuscript is organized as follows. First, MOKE microscopy is used to estimate the surface inclination of a domain wall in the static case. The measurements are carried out on the full length of wires in order to distinguish between long-range effects, i.e. magnetostatic interactions of the domain wall with the ends of the wires, and local effects arising from domain wall pinning. In the next part, we show that microwires with domain wall inclination are characteristic due to the domination of axial stresses. Finally, we perform micromagnetic simulations to ascertain the influence of the axial anisotropy on the domain wall shape. The experimental results are related to previous measurements of domain wall length by induction and optical methods.

\section{Samples and experimental}

Measurements are performed on cylindrical wires produced by the Taylor-Ulitovski method [20]. The fabrication process involves drawing of the molten master alloy with glass coating through a water jet that ensures fast quenching of the as-prepared composite. Furthermore, as a result of different thermal expansion coefficients of the metallic core and glass-coating, very strong stresses are introduced to the wires [21,22]. In our case, a $3 \mathrm{~cm}$ long $\mathrm{Fe}_{77.5} \mathrm{Si}_{7.5} \mathrm{~B}_{15}$ sample with a total diameter (metallic core plus glass thickness) of $36 \mu \mathrm{m}$ is used. The alloy composition is characterized by the absence of a surface shell of domains [23,24]. Microwires of such a composition have been intensively studied due to socalled "unidirectional effect", where the domain wall velocity depends on the orientation of magnetization to which the microwire is magnetized relative to the domain wall movement [25].

The overall schematics of the experiment are shown in Fig. 1. The domain wall inclination is observed by magneto-optical microscopy $[26,27]$ using a Zeiss EC EPIPLAN 50x objective (numerical aperture $N A=0.8$ ), having an aperture large enough to observe a surface of magnetic cylinder, corresponding to angles from the normal up to $\pm 20^{\circ}$ [28]. Observations are carried out in longitudinal $p$-MOKE configuration with the global angle of incidence optimized for the cylindrical surface [28]. The microwire is oriented along the plane of incidence and the magnetic contrast is detected at both sides of the 
cylinder. The change of axial magnetization direction is detected as a characteristic dark and bright MOKE contrast [28]. Thus, the inclined domain wall is identified by the disproportion between the lengths of dark and bright contrasts (Fig. 4b). A detailed explanation of the observed magneto-optical contrasts is provided in section III.II. To assure a reliable estimation of the domain wall inclination, the microwire is rotated up to the angle with the highest surface domain wall length. This is achieved by cutting the microwire into small pieces with a length of $3 \mathrm{~cm}$ and each piece is rotated by $90^{\circ}$. To achieve small additional rotations, the microwire is fixed to a rotating sample holder with diameter of $1 \mathrm{~mm}$ (Fig. 1). The rod is rolled on a double-face adhesive tape with a blackened surface to minimize light reflections from the supporting aluminium stage. It is worth mentioning that the angle of rotation of the sample holder and the angle of rotation of the microwire are equal with respect to the incoming light.

The angular dependence of magnetization loops is measured by alternating gradient force magnetometry (AGFM). The alternating field gradient exerts an alternating force on the sample, proportional to the magnitude of the gradient field and the magnetic moment of the sample. A microwire with a length of $6 \mathrm{~mm}$ is placed on an extension rod attached to a piezoelectric element. The resulting deflection of the extension rod is used to determine the magnetization value along the direction of the applied magnetic field.

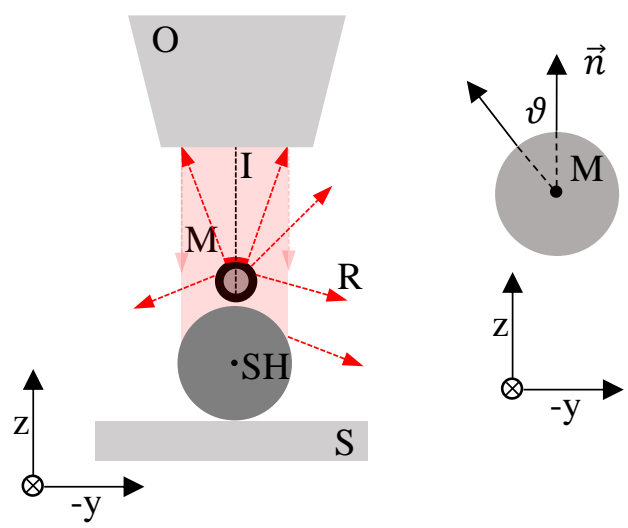

Fig. 1 Schematic illustration of the experimental setup. The microwire $\mathrm{M}$ is fixed to the rotating sample holder $\mathrm{SH}$, which is supported by an $x y$ translational stage $S$ with adhesive tape on top. Incident light $I$ is illustrated by pink colour. Red arrows depict the direction of reflected optical rays $R$. The observations are carried out by an objective $\mathrm{O}$. The position at the surface of the microwire is parametrized by the angle theta.

\section{Results}

\section{III.I Control of a domain wall position}

A key-prerequisite for reliable estimation of the domain wall length is a precise control of the domain wall position. In our experiment, the position of the domain wall is stabilized by two magnetic fields $\mathrm{H}_{1}$ and $\mathrm{H}_{2}$ produced by coils $\mathrm{C} 1$ and $\mathrm{C} 2$ (Fig. 2a). Fig. 2b shows the strength of the resulting magnetic field $\vec{H}=\vec{H}_{1}+\vec{H}_{2}$ as a function of the position for several values of driving current in coil $\mathrm{C} 2$. 
(a)

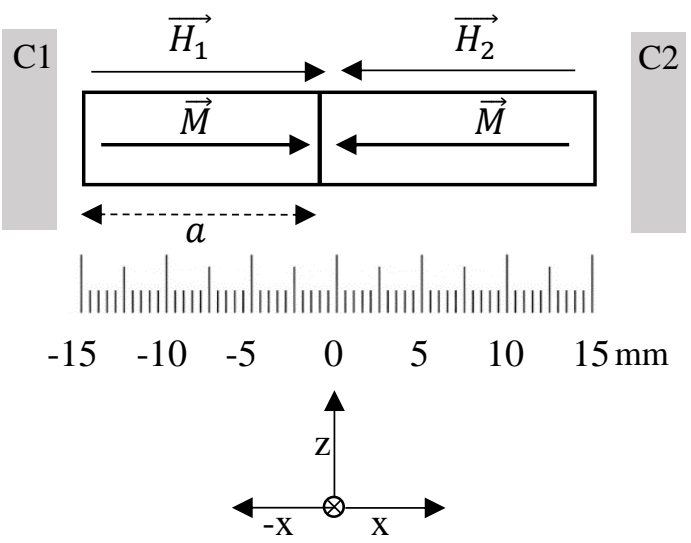

(b)

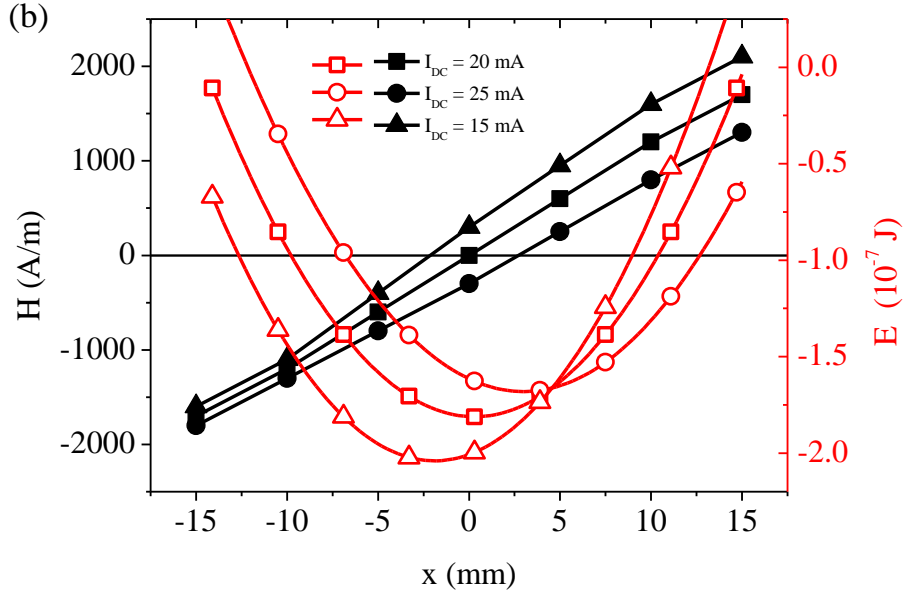

Fig. 2 (a) Schematic illustration of the coil system used for the stabilization of the domain wall. C1 and C2 denote the set of two coils producing the opposite magnetic fields $H_{1}$ and $H_{2}$. (b) Magnetic field $H$ as a function of the position between the two coils. The energy $E$ profile corresponds to the one-dimensional potential well, where the location of the minimum can be simply controlled by adjusting the current in one of the coils.

The energy of a microwire in external magnetic field $H$ is given by

$$
E(a)=-\mu_{0} S \int_{0}^{L} \vec{M} \cdot \vec{H} d x
$$

where $S$ is the cross-sectional surface of a wire, $a$ is the distance of a domain wall from end of a wire, $\vec{H}$ is the local magnetic field (black curves in Fig. 2b) and $\vec{M}$ is the local magnetization. The integration in Eq. 1 is performed over the entire length $L$ of a wire. In line with the general assumption that the microwires under study do not contain surface shell domains, the magnetization $\vec{M}$ has always an axial direction, which simplifies the calculation of the energy $E(a)$. Moreover, we consider that the size of the domain wall is much smaller than the volume of the wire, so the overall energy is mainly given by the contributions from magnetic domains. A plot of Eq. 1 for three different values of the driving electric current is presented in Fig. $2 b$. As seen, for every gradient of the magnetic field, the energy minimum is achieved at the position that coincides with zero local magnetic field. Thus, the system with two opposite magnetic fields applied to the wire with a head-to-head domain wall generates an effective onedimensional potential well. Furthermore, the position of the potential well can be precisely controlled by the electric currents in the magnetic coils (Fig. 2b), which provides a smooth and precise control of the domain wall position.

\section{III.II Optimization of magneto-optical contrast}

In MOKE microscopy, the detection of magnetic contrast is based on the change of a light intensity in an optical polarization microscope due to interaction of a polarized light with the surface magnetism. The contribution of the cylindrical sample geometry to the overall magneto-optical effects has been 
discussed in our previous works [28]. It was found that the specific optical features of thin cylinders, like the spatial orientation of planes of incidence or mixed local polarizations, must be taken into account for a correct estimation of the magneto-optical contrast. Furthermore, the strength of the magneto-optical contrast of thin magnetic cylinders depends on several other parameters like the global angle of incidence (defined as the angle between normal at the top of the wire and the incoming light), which is limited by the numerical aperture of the used objective. In agreement with the formalism introduced in [28] and using the same coordinate system, the intensity of the reflected light from the cylindrical surface in the longitudinal p-MOKE configuration and using an optical compensator by rotating a quarter-wave plate, can be expressed as

$$
I=|\vec{S} \cdot \overleftrightarrow{R} \cdot \overleftrightarrow{Q} \cdot \vec{P}|^{2}
$$

where $\vec{S}$ is the global S polarization, $\overleftrightarrow{R}$ is the reflection matrix that consists of Fresnel coefficients, $\vec{P}$ is the global P polarization [28] and $\overleftrightarrow{Q}$ is the matrix of compensator given by

$$
\overleftrightarrow{Q}=\left[\begin{array}{cc}
\cos ^{2} \varphi+i \sin ^{2} \varphi & (1-i) \sin \varphi \cos \varphi \\
(1-i) \sin \varphi \cos \varphi & \sin ^{2} \varphi+i \cos ^{2} \varphi
\end{array}\right]
$$

where $\varphi$ is the angle between the slow axis of the compensator and the $S$ polarization. A plot intensity using Eq. 2 for a cylinder with axially magnetized surface is presented in Fig. 3(a). As seen, for any rotation of the compensator, the profile consists of two intensity maxima, similarly to the case without compensator [28]. On the other hand, even a slight rotation of the compensator increases the light intensity at one side of the circular wire at the expense of the other side. The change of light intensity due to interaction with surface magnetization is very small and it has the same sign at both sides of a cylinder (blue and cyan curves in Fig. 3(a)).
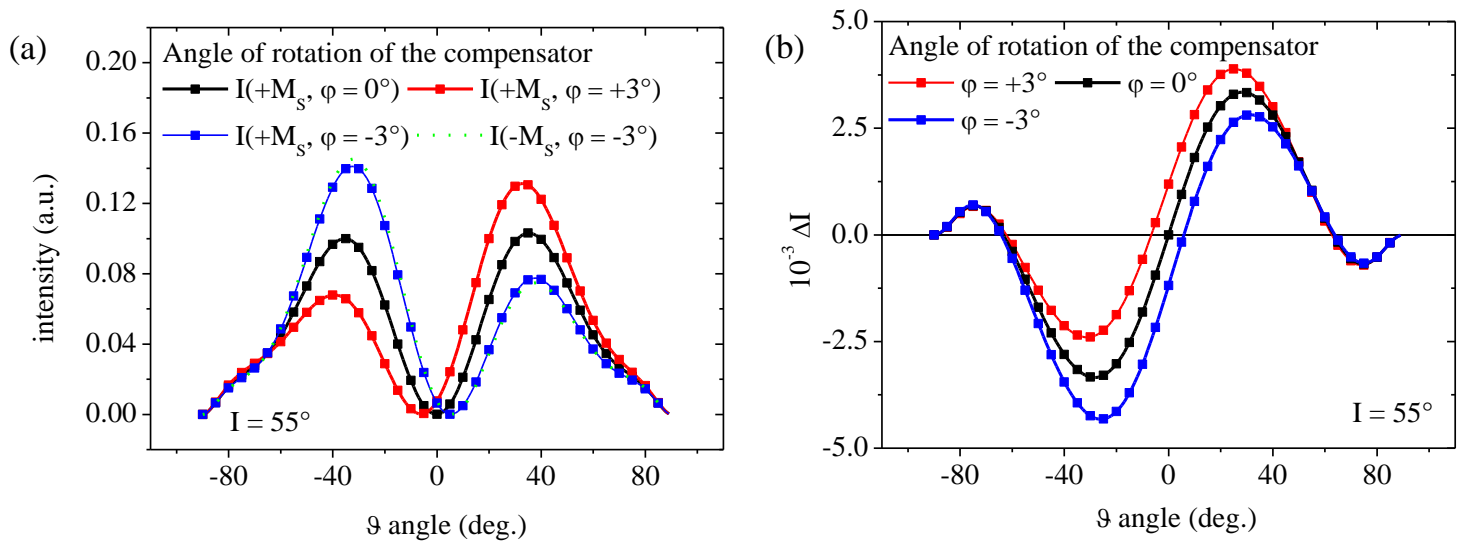

Fig. 3. (a) Intensity of reflected light as a function of the rotation of compensator ( $\lambda / 4$ plate) by an angle $\varphi$. Calculations are carried out for a compensator placed between the sample and polarizer in a longitudinal p-MOKE 
configuration. (b) The magneto-optical contrast as a function of the rotation of the compensator. The angle theta describes the position on the cylinder surface [28] (Fig. 1).

For magnetic cylinders with axial magnetization at the surface, the maximum magnetic contrast obtained by differential image subtraction is simply proportional to the change of light intensity given by

$$
\Delta I=I\left(+M_{S}, \varphi\right)-I\left(-M_{S}, \varphi\right),
$$

where $M_{s}$ is the saturation magnetization at the surface and $\varphi$ is the rotation of compensator from the $S$ polarization. The intensity difference $\Delta /$ obtained from Eq. 4 and Eq. 3 for several values of the angle $\varphi$ is shown in Fig. 3(b). As seen, a change of surface magnetization direction results in a characteristic black and white contrast that is typical for cylinders with spontaneous magnetization oriented along the principal axis (see also Fig. 4(b)). The contrast was explained by the local mixed polarization of the light [28]. Due to the spatial distribution of the local planes of incidence, the linear polarization of the incident light can be decomposed into the perpendicular (i.e. local s) and parallel (local p) components with respect to the local plane of incidence [28]. The opposite contrast is given by the dominant contribution from the local s polarization that has opposite sign at both sides of the cylinder. As seen in Fig. 3(b), the rotation of a compensator suppresses the contrast at one side and enhances it at the other. Fig. 4 compares the profile of the magneto-optical contrast detected by MOKE microscopy for several rotations of the compensator. As seen in Fig. 4(b), the correct rotation of the compensator is a key for obtaining well-balanced magneto-optical contrasts at both sides of the cylindric microwire.

(a)

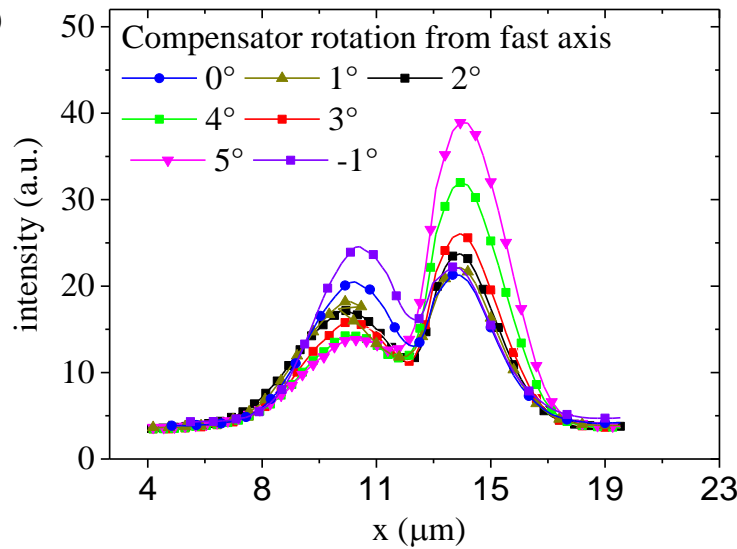

(b)
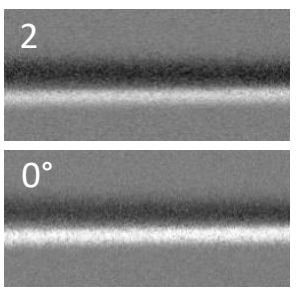

$-4^{\circ}$

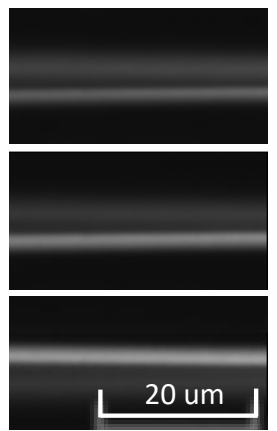

Fig. 4. (a) Light intensity as a function of the compensator rotation in MOKE microscopy. Experiments are carried out in the longitudinal p-MOKE configuration. (b) The magneto-optical contrast (left column) and corresponding optical intensity image (right column) with varying rotation of a compensator.

\section{III.III Observations of domain wall by MOKE microscopy}

First observations of domain wall shapes were performed in the central region of the microwire. The measured surface domain wall length is displayed in Fig. 5(a). Figs. 5(b) and 5(c) show typical magnetooptical images of domains stabilized by two opposite magnetic fields. Generally, the length of the characteristic contrasts differs at both sides of the cylinder, which implies a tilted orientation of the domain wall. It is worth mentioning that the magnetic field gradients of up to $2 \mathrm{kA} / \mathrm{m}^{2}$ are not strong 
enough to change the apparent domain wall inclination. Thus, the observed shape of the domain wall does not result from the interaction with the external magnetic field, but it appears to be an intrinsic feature of the domain wall. This assumption is additionally confirmed by the fact that the apparent domain wall orientation is strongly correlated to the rotation of the microwire around its principal axis (Fig. 5(a)). The domain wall rotates together with the mechanical rotation of the microwire and externally applied fields with perpendicular orientation changes neither the characteristic orientation nor the length of the domain wall. In this work, the length of a domain wall is estimated by precise positioning the microwire to a $90^{\circ}$ position, where the surface length is higher than the field of view of the used microscope objective. Then, the apparent surface length of the domain wall is obtained indirectly by measurements of the distance between the ends of the stripes of different contrast, when the domain wall is moved out from the field of view.

(a)

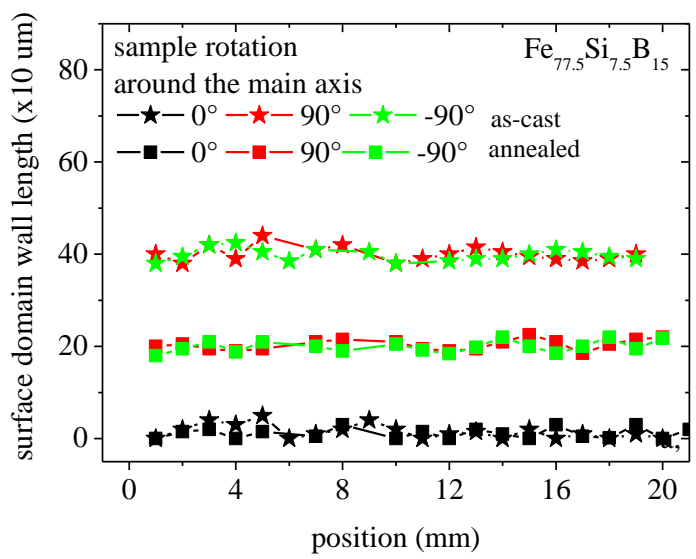

(b)

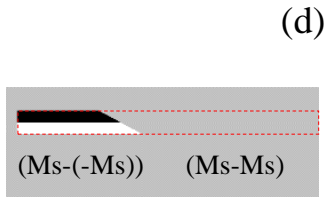

(c)

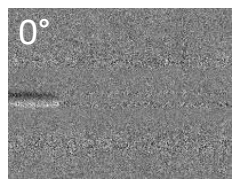

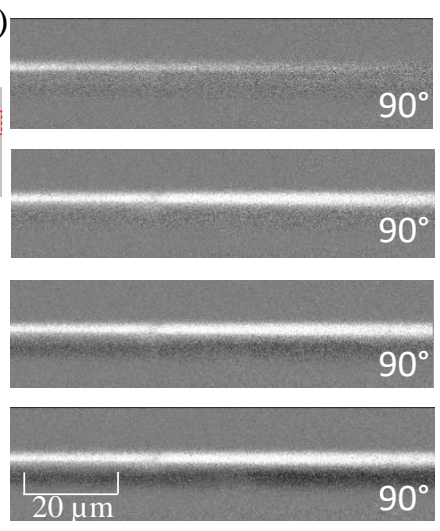

Fig. 5 (a) The apparent domain wall length as a function of the position along an as-cast (star symbol) and an annealed (square symbol) FeSiB microwire. (b) Schematic illustration of the black-and-white magneto-optical contrast obtained by the differential imaging technique. Red dashed lines depict the visible part of the wire. (c) Magneto-optical image of a domain wall in the as-cast sample obtained by MOKE microscopy. (d) Magneto-optical image of a domain wall in several positions with respect to the field of view. The domain wall is moved from the left to the right. The rotation angle of $90^{\circ}$ is measured from the position, where the apparent surface domain length is zero (Fig. 5(c)).

While the local defects are the origin of domain wall nucleation at high uniaxial magnetic fields [28], the interplay between defects and the local shape of domain wall has been a matter of discussion for years [30]. Fig. 5(a) shows the surface domain wall length estimated at different positions along the length of wire. Variations of the local domain wall length do not exceed 10 percent and the local changes are barely detectable for small domain wall displacements. Owing to the amorphous origin of the sample, the potential landscape of a domain wall is not spatially homogeneous. However, variations of the surface domain wall length observed in our experiment are not as high as for the local map of nucleation fields [28]. This result indicates that the surface domain wall pinning is less important than the pinning in the bulk. This finding is not surprising, as the bulk pinning is obviously the dominant contribution to 
the overall domain wall pinning in wires with large diameter [31]. Moreover, the surface domain wall length does not change remarkably neither close to the end of wire $(7 \mathrm{~mm})$. From that result, we infer that the domain wall inclination does not originate from the interaction with uncompensated magnetic charges, as it was observed in shorter wires [18], where the magnetostatic contribution to the overall domain wall energy is more significant. It is inherent to the properties of the microwire.

From the spatial distribution of the surface domain wall lengths in the annealed sample in Fig. 5(a) it can be seen that the maximum domain wall length is decreased significantly as compared to the as-cast wire. As in the as-cast state, variations of surface domain wall length do not exceed 10 percent and neither orientation nor tilting are changed in regions close to the ends of the wire. The observed increase of a surface domain wall length may be associated with the reduction of internal mechanical stresses during the annealing process, which through magnetoelastic coupling influences the overall magnetic anisotropy distribution in the microwire.

\section{III.IV Magnetometry measurements}

Stress-induced magnetic anisotropy is one of the most important anisotropies that determine the domain structure of as prepared microwires. As a result of the local cooling during fabrication process, strong mechanical stresses with axial, radial and azimuthal orientation may be introduced to the sample [21]. It was shown in previous works [32] that the magnitude and spatial distribution of mechanical stresses is very sensitive to the preparation conditions. In order to quantify the amplitude of internal mechanical stresses of our sample, we apply a modified Stoner-Wohlfarth model. The magnetic energy density $E_{m a g}$ of uniaxial ferromagnets placed in magnetic field $H_{\text {ext }}$ is expressed as

$$
E_{m a g}=K_{U} \sin ^{2}\left(\phi_{S}-\varphi_{S}\right)-\mu_{0} M_{S} H_{e x t} \cos \phi_{S}
$$

where $M_{S}$ is the saturation magnetization, $\mu_{0}$ is the vacuum permeability, $K_{U}$ is the constant of uniaxial magnetic anisotropy, $\varphi_{S}$ is the angle between the magnetic field direction and the easy axis of magnetization, and $\phi_{S}$ is the angle between the magnetic field direction and magnetization. The energy minimum is then given by

$$
K_{U}\left(\sin 2\left(\phi_{S}-\varphi_{S}\right)+2 \frac{H_{\text {ext }}}{H_{K}} \sin \phi_{S}\right)=0
$$

where $H_{K}=2 K_{U} / \mu_{0} M_{S}$ is the anisotropy field. In our work, comparison of the uniaxial Stoner-Wohlfarth model with the magnetization process of the microwire has been carried out in four successive steps: (1) the descending branch of a magnetization loop is measured at certain angle $\varphi_{S}$, (2) Eq. (6) is used to calculate the field dependence of axial magnetization. The angle $\varphi_{S}$ is measured by a high-resolution camera equipped with a zoom objective. The saturation magnetization $M_{S}$ is obtained from step (1). (3) The uniaxial anisotropy field value $H_{K}$ is determined by the least square method. (4) Steps (1)-(3) are repeated for various angles of $\varphi_{S}$. 
(a)

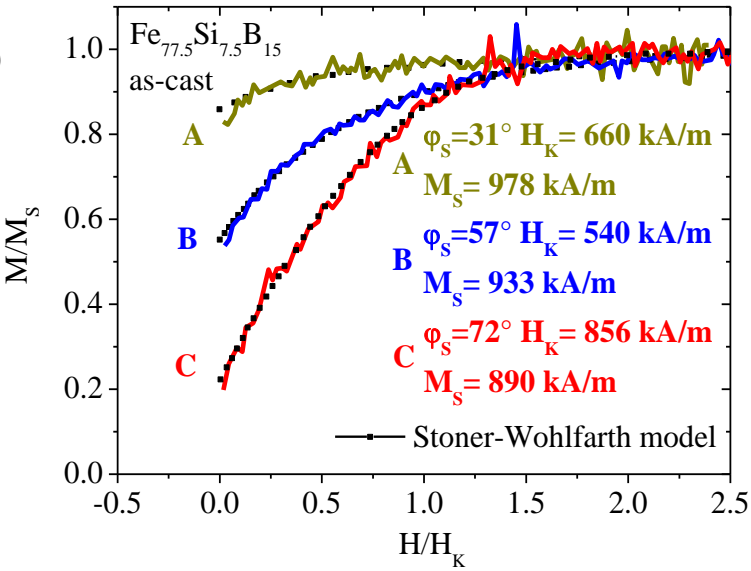

(b)

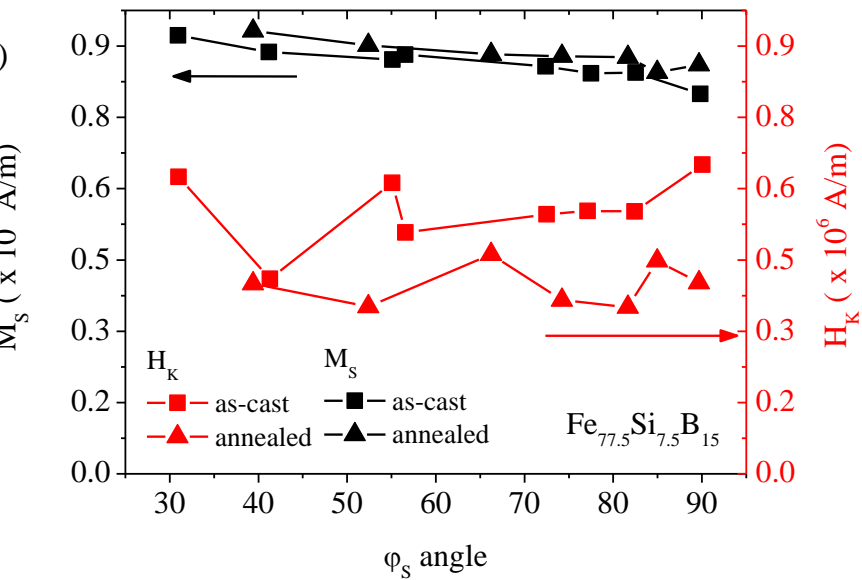

Fig. 6 (a) Comparison of the Stoner-Wohlfarth model (red curves) to the experiment (color curves). (b) Plot of the saturation magnetization and the anisotropy field. Data are obtained from fitting the ascending branches of hysteresis loops before and after annealing.

Exemplary fits of the Stoner-Wohlfarth model for the as-cast sample are presented in Fig. 6(a). A good agreement of the experiment with the Stoner-Wohlfarth model is obtained for each angle of rotation. However, Fig. 6(b) shows that the fitted values of the anisotropy field and the saturation magnetization are slightly varying with sample rotation $\varphi_{S}$. We infer from this result that the studied material has spatially non-uniform magnetization directions. This agrees with general assumptions, considering a closure domain wall structure at the ends of wire [21]. The density of the uniaxial magnetic anisotropy $K_{U}$ from shape $K_{\text {demag }}$ and magnetoelastic anisotropy $K_{\text {stress }}$ can be expressed as

$$
K_{U}=K_{\text {demag }}+K_{\text {stress }}=\frac{\mu_{0} M_{S} H_{K}}{2} .
$$

Using an average value of the magnetic anisotropy field $H_{K}=591 \mathrm{kA} / \mathrm{m}$ and the saturation magnetization $M_{S}=913 \mathrm{kA} / \mathrm{m}$, Eq.(7) gives $K_{U}=3.4 \times 10^{5} \mathrm{~J} / \mathrm{m}^{3}$. The demagnetizing field of a long cylinder is given by $H_{\text {demag }}=M_{S} / 2$, so the contribution of shape anisotropy to the corresponding density of uniaxial magnetic anisotropy can be evaluated as $H_{\text {demag }}=2.6 \times 10^{5} \mathrm{~J} / \mathrm{m}^{3}$. Thus, the density of uniaxial magnetic anisotropy arising from magnetoelastic coupling is given by $K_{\text {stress }}=0.8 \times 10^{5} \mathrm{~J} / \mathrm{m}^{3}$, which agrees with previous results [33]. The value of the magnetoelastic anisotropy energy density is surprisingly high. Referring to pure iron [34], the magnetoelastic anisotropy energy density is lower, but of comparable magnitude. It can be expected that such high axial magnetic anisotropy influences the domain wall shape as well. 
Fig. 7(a) shows ascending branches of the magnetization loops of a thermally annealed FeSiB microwire. The data are used to the extract fitting parameters presented in Fig. 6(b). As seen, the average value of the fitted anisotropy field $H_{K}=420 \mathrm{kA} / \mathrm{m}$ is here slightly smaller as compared to the as-cast sample. The value of magnetic anisotropy field in the annealed microwires is close to $H_{\text {demag }}$, so the magnetoelastic anisotropy has almost vanished. This can be attributed to the relaxation of the internal stresses during the annealing procedure.
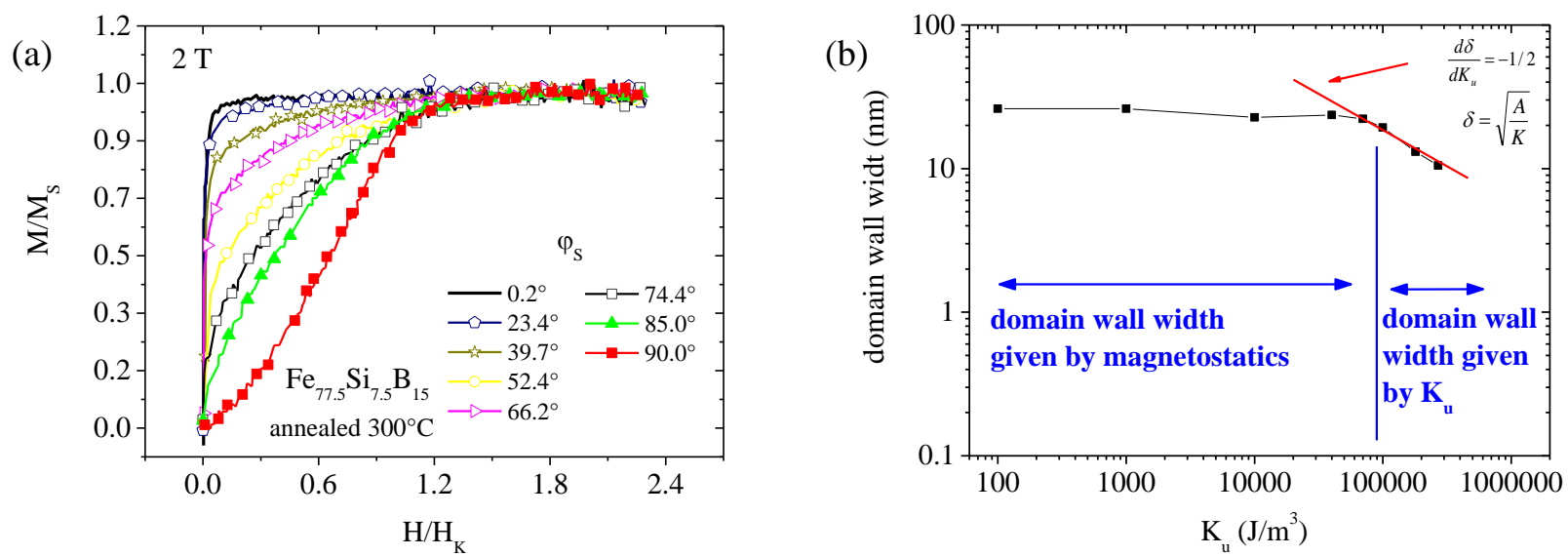

Fig. 7 (a) Ascending branches of magnetization loops measured by AGFM for various angles between the field direction and the easy axis of anisotropy orientation. The maximum of applied field is 2T. (b) Domain wall width as a function of uniaxial magnetic anisotropy. The calculation of the domain wall width is done by the Thiele formula (Eq. 8).

\section{III.V Micromagnetic model}

The micromagnetic simulations were performed by the Object Oriented Micro Magnetic Framework (OOMMF) software [35]. In order to show that the tilted orientation of a domain wall is not a direct consequence of cylindrical shape of the sample, simulations are carried out on a thin rod with a square cross-section. A finite difference method is employed to simulate the spontaneous magnetization of individual nanowires with a $200 \mathrm{~nm}$ long side of square cross-section and a length of $2000 \mathrm{~nm}$. The wires are discretized into uniform cubic meshes with a size of $5 \mathrm{~nm} \times 5 \mathrm{~nm} \times 5 \mathrm{~nm}$. The magnetization is evolved from an initial configuration consisting of two opposite magnetic domains with a transient region with perpendicular magnetization. The domain wall shape is obtained by minimization of the total energy for several values of the uniaxial magnetic anisotropy $K_{U}$. The material parameters for simulation include $M_{S}=8.4 \times 10^{5} \mathrm{~A} / \mathrm{m}$ and $A=13 \times 10^{-12} \mathrm{~J} / \mathrm{m}$, where $M_{S}$ is the saturation magnetization and $A$ is the exchange constant. The $M_{S}$ value is extracted from fits of the Stoner-Wohlfarth model at $\varphi_{S}=85^{\circ}$, where the saturation magnetizations of annealed and as-cast samples are equal (Fig. 6b). The exchange constant has a typical value for iron-based alloys [36].

Figure 8 shows the simulated magnetization distribution of an individual nanowire with varying domain wall shape as a function of the uniaxial magnetic anisotropy. Starting from Fig. 8(a), a screw-like domain wall is obtained at zero axial magnetic anisotropy. With further increase of the axial anisotropy, the number of turns of domain wall decreases (Fig. 8(b)-(c)). When the anisotropy constant exceeds $K_{U}=$ $1.0 \times 10^{5} \mathrm{~J} / \mathrm{m}^{3}$, the uniaxial magnetic anisotropy dominates, and the domain wall becomes planar with 
a tilted orientation at the surface. It is worth mentioning that the domain wall tilting is clearly visible in all magnetic domain images. The micromagnetic calculations reveal distinct domain wall patterns as a result of the system attempts to reduce its energy through twisting and formation of tilted magnetic structures orthogonally to the main axis.

(a)

(b)

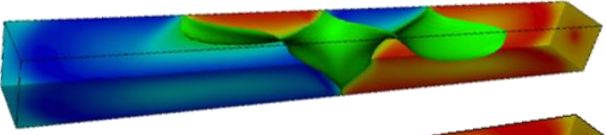

(c)

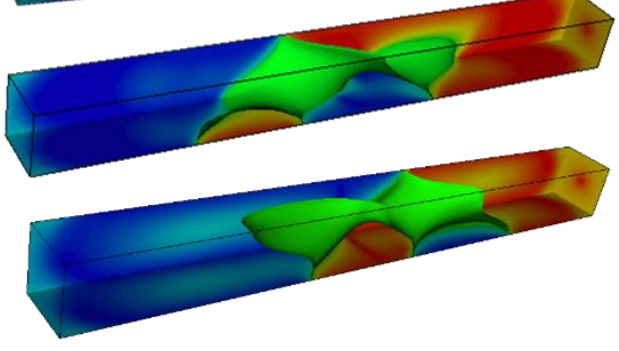

(d)

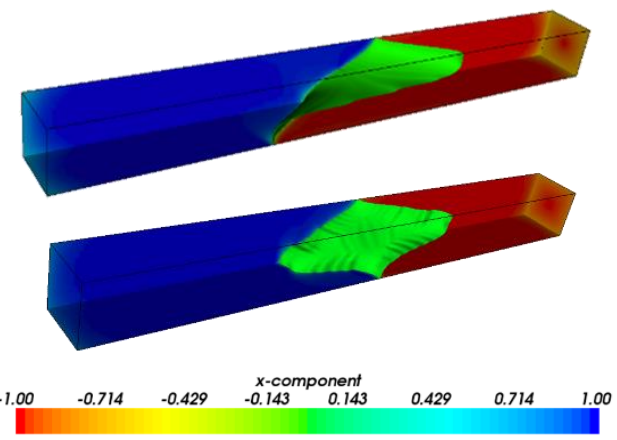

Fig. 8 Magnetic component along the wire length obtained from micromagnetic simulations displaying the domain wall shape as a function of the axial magnetic anisotropy. The axial $\mathrm{x}$-component of magnetization is coded by the color scale. (a) $K_{u}=0 \mathrm{~J} / \mathrm{m}^{3}$, (b) $K_{u}=1.1 \times 10^{2} \mathrm{~J} / \mathrm{m}^{3}$, (c) $K_{u}=1.1 \times 10^{4} \mathrm{~J} / \mathrm{m}^{3}$, (d) $K_{u}=1.1 \times 10^{5} \mathrm{~J} / \mathrm{m}^{3}$, and (e) $K_{u}=2.75 \times 10^{5}$ $\mathrm{J} / \mathrm{m}^{3}$. The nanowire has a length of $2000 \mathrm{~nm}$ and a width of $200 \mathrm{~nm}$. The orientation of each image is slightly changed to make the shape of a domain wall better visible.

In order to further clarify the origin of a domain wall tilting presented in Fig. 8, the domain wall width is calculated using the Thiele formula [37,38]

$$
\frac{2 s}{\Delta}=\int\left(\frac{d m}{d x}\right)^{2} d x d y d z,
$$

where $S$ is the cross-sectional area, $\Delta$ is the effective width and the integral is applied for the whole volume of nanowire. Fig. 7 (b) shows the anisotropy dependence of the calculated domain wall width. For small values of the anisotropy constant, the domain wall width remains constant. An increase in the uniaxial magnetic anisotropy does not result in a smaller domain wall width. Thus, the domain wall width in this region is governed by magnetostatics. On the other hand, if the anisotropy constant exceeds $K_{U}=1 \times 10^{5} \mathrm{~J} / \mathrm{m}^{3}$, the width of the domain wall is well approximated by a square-root formula (Fig. $7(b)$ ), which confirms a dominant role of the uniaxial magnetic anisotropy on the domain wall inclination observed in this region. Referring to the anisotropy fields of microwires (Fig. 6b), the $K_{U}$ value of real samples is between $2.6 \times 10^{5} \mathrm{~J} / \mathrm{m}^{3}$ (annealed state) and $3.4 \times 10^{5} \mathrm{~J} / \mathrm{m}^{3}$ (as-cast state). In both cases, the magneto-optical imaging confirmed a tilted orientation of a domain wall at the surface.

Overall, the occurrence of a tilted domain wall relates to the reduction of magnetostatic energy and magnetic charge density through the increase of the effective wall length. In several systems, the presence of a high uniaxial magnetic anisotropy may lead to formation of a periodic zig-zag domain wall pattern that has been investigated both experimentally $[39,40]$ and theoretically [41] in thin films. The presented micromagnetic analysis shows that the high uniaxial magnetic anisotropy leads to an inclined domain wall orientation in individual wires as well. 


\section{Conclusions}

In summary, we have studied the characteristics of domain wall inclination in amorphous glass-coated microwires with positive magnetostriction. Magneto-optical imaging showed that the apparent orientation of a domain wall does not significantly change its tilting orientation, also not close to the ends of wire. The tilting angle decreases remarkably with the release of mechanical stresses by thermal annealing. A comparison of magnetization loops with the Stoner-Wohlfarth model reveals that microwires with tilted domain walls are characteristic to mechanical stresses with dominant axial orientation in the wires. Finally, micromagnetic simulations are used to further prove that the tilted domain wall orientation can be achieved by sufficiently high uniaxial magnetic anisotropy.

\section{Data availability statement}

The data that support the findings of this study are available from the corresponding author upon request.

\section{Acknowledgements}

This work was supported by the bilateral Slovak-French project APVV SK-FR2017-0024 (PHC Stefanik 40571TE), Slovak grant Agency APVV-17-0184, and Slovak grant Agency VEGA 1/0195/18. The help of Thibaut Devolder for valuable comments and introduction to the AGFM technique is gratefully acknowledged. K.R. acknowledges the French embassy in Slovakia for granting a Bourse du gouvernement français (BGF).

\section{References}

[1] M. Smelko, M. Spodniak, K. Semrad, et al. Aeronautical composite construction monitoring by magnetic microwires, NTAD 2018 - 13th International Scientific Conference - New Trends in Aviation Development, Proceedings 8551677, 128 (2019).

[2] S. S. P. Parkin, M. Hayashi, L. Thomas, Magnetic Domain-Wall Racetrack Memory, Science 11, 190 (2008).

[3] D. Praslička, M. Smelko, J. Blazek, J. Hudak, P. Lipovsky, N. Flachbart, Advanced method for magnetic microwires noise specification, Acta Physica Polonica A. 126, 86-87 (2014).

[4] M. Al. Bahri, et al., Staggered magnetic nanowire devices for effective domain-wall pinning in racetrack memory, Phys. Rev. App. 11, 024023 (2019).

[5] S. Bhatii, Ch. Ma, X. Liu, and S.N. Piramanayagam, Realization of Energy Harvesting Based on Stress-Induced Modification of Magnetic Domain Structures in Microwires, IEEE Trans. Magn. 55, 2301107 (2019).

[6] K. Richter, M. Mawass, A. Krone, et al, Local domain wall velocity engineered by tailored potential landscapes in ferromagnetic rings, Phys. Rev. Appl. 5, 024007 (2016).

[7] F. Nasirpouri, S. M. P. Sattri, C. Bran, et al, Geometrically designed domain wall trap in tri-segmented nickel magnetic nanowires for spintronics devices, Sci. Rep. 9, 9010 (2019). 
[8] J. Pablo-Navarro, D. Sanz-Hernández, C. Magén, A. Fernández-Pacheco, and J. M. Teresa, Tuning shape, composition and magnetization of 3D cobalt nanowires grown by focused electron beam induced deposition (FEBID), J. Phys. D 50, 18LT01 (2017).

[9] M. Vazquez, A. Zhukov, Magnetic properties of glass-coated amorphous and nanocrystalline microwires, J. Magn. Magn. Mater. 160, 223 (1996).

[10] S. Corodeanu, H. Chiriac, A. Damian, N. Lupu, et al, Field and current controlled domain wall propagation in twisted glass-coated magnetic microwires, Sci. Rep. 9, 5868 (2019).

[11] J. Onufer, J Ziman, P. Duranka, M. Kladivova, The influence of annealing on domain wall propagation in bistable amorphous microwires with unidirectional effect, Phys. B: Condensed matter 540, 58 (2018).

[12] K. Richter, R. Varga, G. Badini-Confalonieri, M. Vázquez, The effect of transverse field on fast domain wall dynamics in magnetic microwires. Appl. Phys.Lett. 96, 182507 (2010).

[13] J. Olivera, M. L. Sanchez, V. M. Prida, R. Varga, V. Zhukova, A. P. Zhukov, B. Hernando, Temperature Dependence of the Magnetization Reversal Process and Domain Structure in FeNiSiB Magnetic Microwires, IEEE Trans. Magn. 44, 3949 (2008).

[14] M. Smelko, J. Kravcak, D. Praslicka, K. Draganova, Impact of modified endings on oise characteristics of Fe-based glass-coated microwires, Journal of electrical engineering-elektrotechnicky casopis 66, 30 (2015).

[15] P. Lipovský, A. Čverha, J. Hudák, J. Blažek, D. Praslička, Noise Characteristics of Microwire Magnetometer, Acta Phys. Pol. A. 126, 384 (2014).

[16] K. Richter, R. Varga, A. Zhukov, Influence of the magnetoelastic anisotropy on the domain wall dynamics in bistable amorphous wires, J. Phys.: Condensed Matter 24, 296003 (2012).

[17] L. V. Panina, M. Ipatov, V. Zhukova, A. Zhukov, Domain wall propagation in Fe-rich amorphous microwires, Physica B 407, 1442 (2001).

[18] P. A. Ekstrom, and A. Zhukov, Spatial structure of the head-to-head propagating domain wall in glass coated FeSiB microwire, Journal of Physics D: Applied Physics 43, 205001 (2010).

[19] O. Vahovsky, R. Varga and K. Richter, Experimental method for surface domain wall shape studies in thin magnetic wires, J. Magn. Magn. Mater. 483, 266 (2019).

[20] H. Chiriac, T.-A. Óvári, M. Tibu, Effect of surface domain structure on wall mobility in amorphous microwires, J. Appl. Phys. 105, 07A310 (2009).

[21] H. Chiriac, et al, Amorphous glass-covered magnetic wires: Preparation, properties, applications, Prog. Mater. Sci. 40, 333 (1996).

[22] A. Talaat, V. Zhukova, M. Ipatov, J. M. Blanco, J. Gonzalez, and A. Zhukov, Impact of Stress Annealing on the Magnetization Process of Amorphous and Nanocrystalline Co-Based Microwires, Materials 12, 16 (2009).

[23] J. Olivera, M. L. Sanchez, V. M. Prida, R. Varga, V. Zhukova, A. P. Zhukov, B. Hernando, Temperature Dependence of the Magnetization Reversal Process and Domain Structure in FeNiSiB Magnetic Microwires, IEEE Trans. Magn. 44, 3946 (2008).

[24] H. Chiriac, T.-A. Ovari, Magnetic properties of amorphous glass-covered wires, J. Magn. Magn. Mater. 249, 46 (2002). 
[25] J. Onufer, J. Ziman, M. Kladivova, Unidirectional effect in domain wall propagation observed in bistable glass-coated microwire, Journal of Magnetism and Magnetic Materials 396, 313 (2015).

[26] J. McCord, Progress in magnetic domain observation by advanced magneto-optical microscopy (Topical Review), Journal of Physics D: Applied Physics 48, 333001 (2015).

[27] A. Stupakiewicz, A. Chizhik, M. Tekielak, A. Zhukov, J. Gonzalez, A. Maziewski, Direct imaging of the magnetization reversal in microwires using all-MOKE microscopy, Rev. Sci. Instrum. 85, 103702 (2014).

[28] K. Richter, A. Thiaville, R. Varga, Analytical calculation and observation of mageto-optical contrast of magnetic cyllinders, Phys. Rev. B 96, 064421 (2017).

[29] M. Ipatov, V. Zhukova, J. Gonzalez, and A. Zhukov, Annealing effect on local nucleation fields in bistable microwies, Phys. Status Solidi 208, 549 (2011).

[30] A. Jimenez, R. P. Del Real, and M. Vazquez, Controlling depinning and propagation of single domain-walls in magnetic microwires, J. Phys. Condens. Matter 86, 113 (2013).

[31] T. Chen, M. Erickson, P. Crowell, C. Leighton, Surface roughness dominated pinning mechanism of magnetic votices in soft ferromagnetic films, Phys. Rev. Lett. 109, 097202 (2012).

[32] H. Chiriac, T. A. Ovari, and Gh. Pop, Internal stress field distribution in glass-covered amorphous magnetic wires, Phys. Rev. B 52, 10104 (1995).

[33] O.I. Aksenov, N. Orlova, et al., Stress state effect on the magnetic properties of amorphous microwires, J. Magn. Magn. Mater. 495, 165878 (2020).

[34] R. Carey and E. D. Isaac, Magnetic domains and techniques for their observation, English Universities Press Limited, 1966 London, p.67.

[35] M.J. Donahue and D.G. Porter, OOMMF User's Guide, Version 1.0, Interagency Report NISTIR 6376, National Institute of Standards and Technology, Gaithersburg, MD (1999).

[36] I. Purnama, J.H. Moon, Ch. You, Eigen damping constant of spin waves in ferromagnetic nanostructure, Sci. Rep. 9, 13226 (2019).

[37] A. A. Thiele, Steady-state motion of magnetic domains, Phys. Rev. Lett. 30, 230 (1973).

[38] B. Hillebrands and A. Thiaville, Spin Dynamics in Confined Magnetic Structures III, (Springer-Verlag Berlin Heidelberg 2006).

[39] E.J. Hsieh, R.F. Soohoo, Configuration of head-to-head domain walls in thin layers, AIP Conf. Proc. 5, 727 (1971).

[40] C. C. Shir, Micro-magnetic structures of planar charged walls, J. Appl. Phys. 50, 2270 (1979).

[41] R.F. Soohoo, Micromagnetics of zigzag head-on domain walls, J. Magn. Magn. Mater. 35, 261 (1983). 


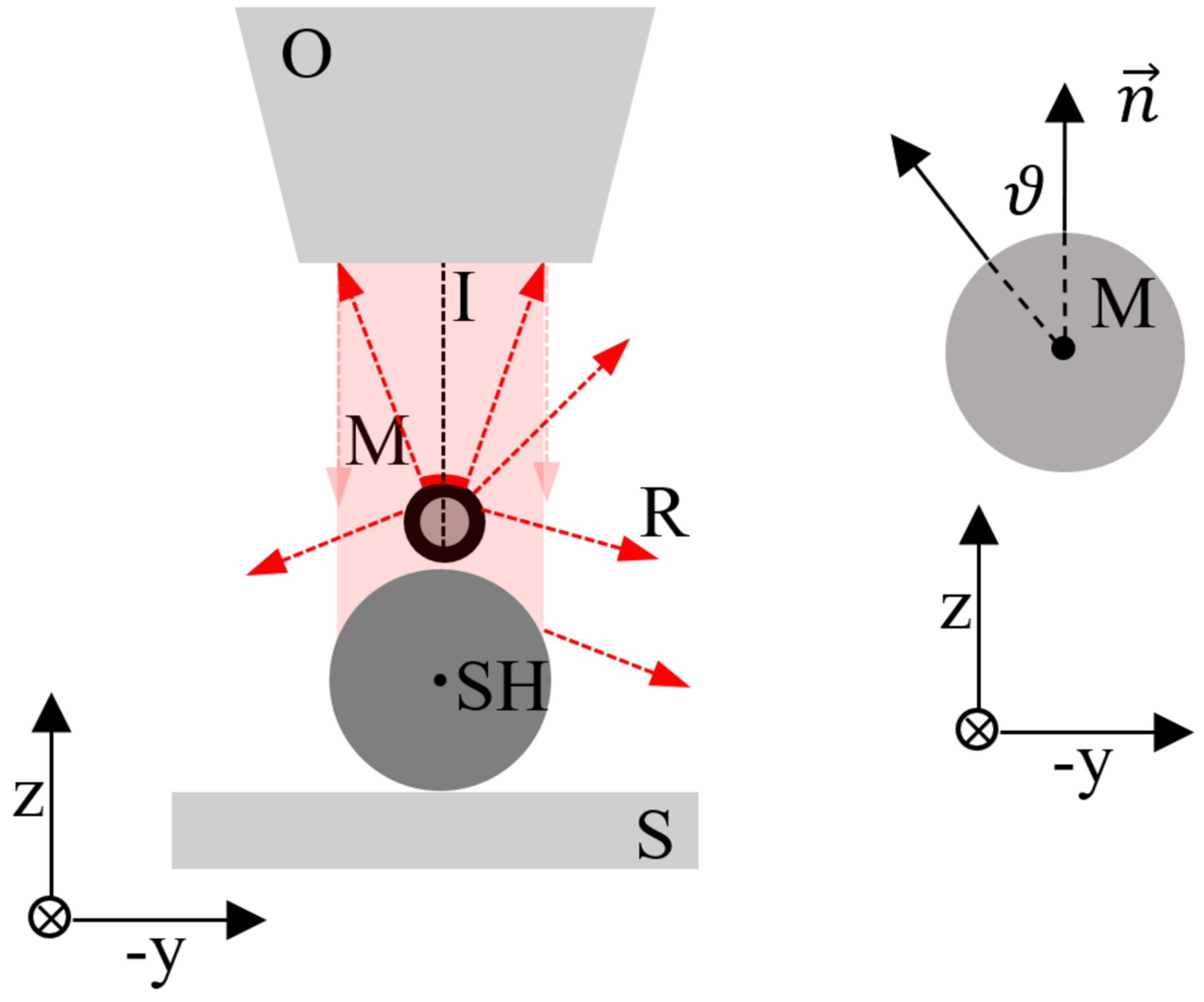




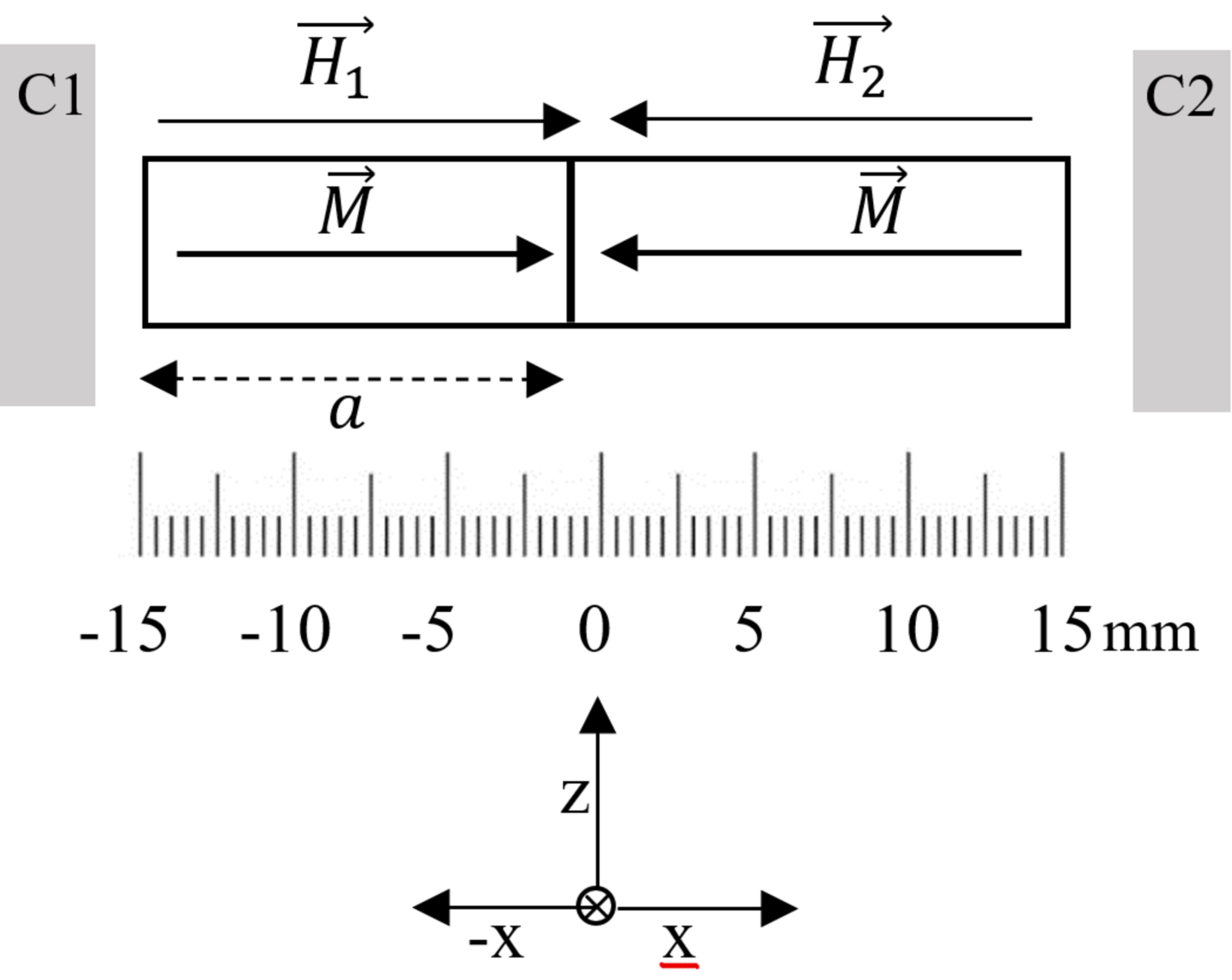




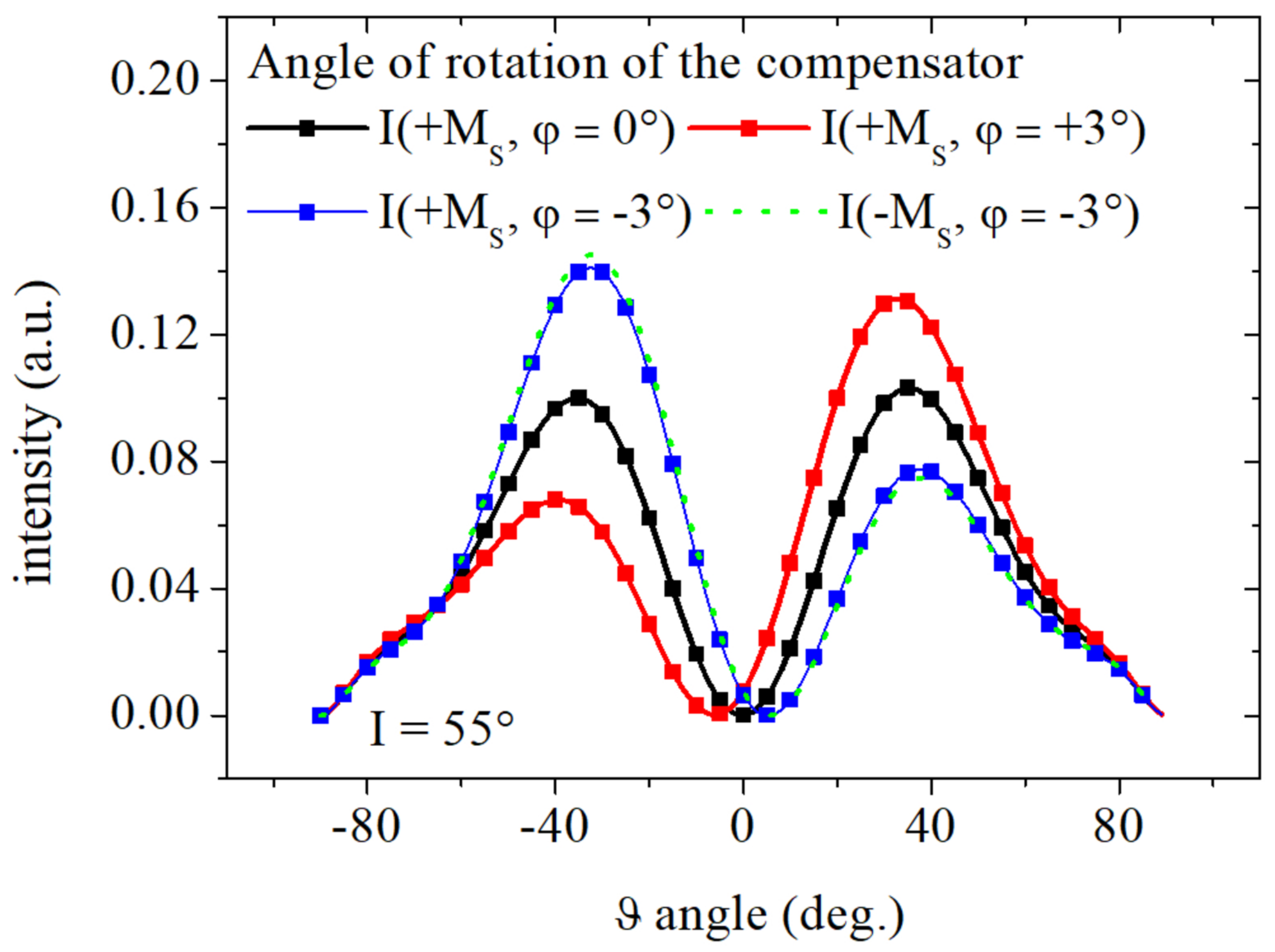




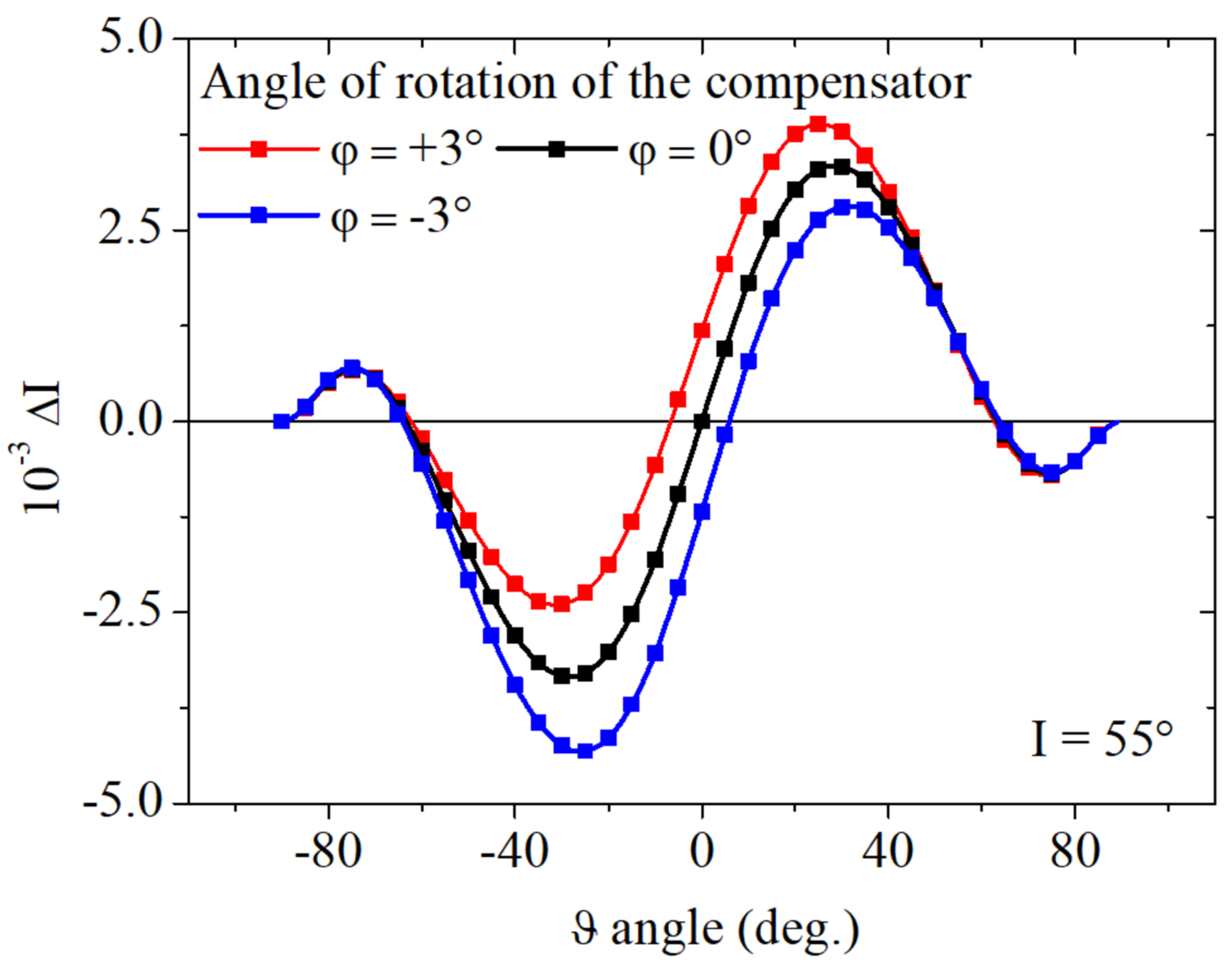




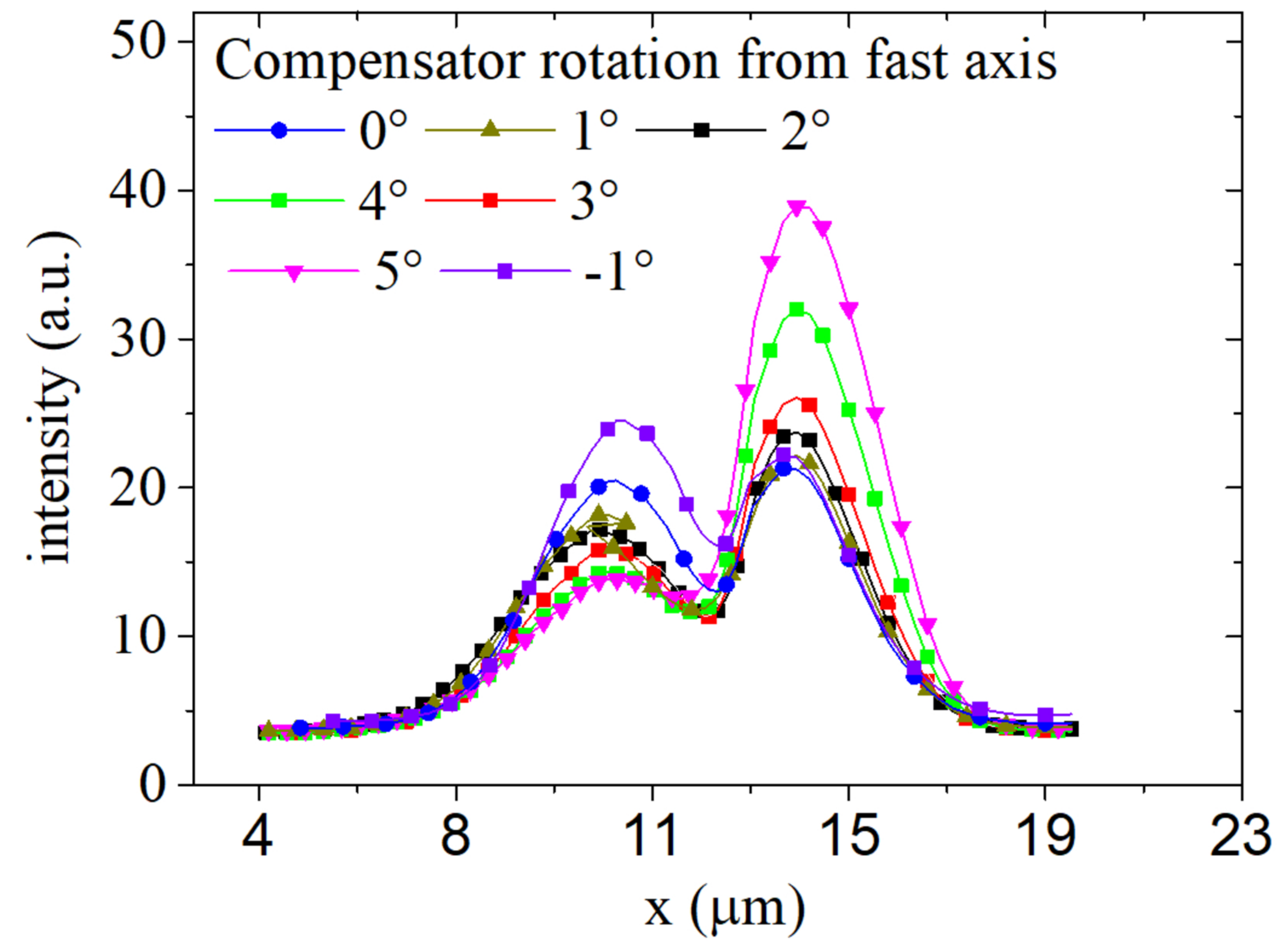


2

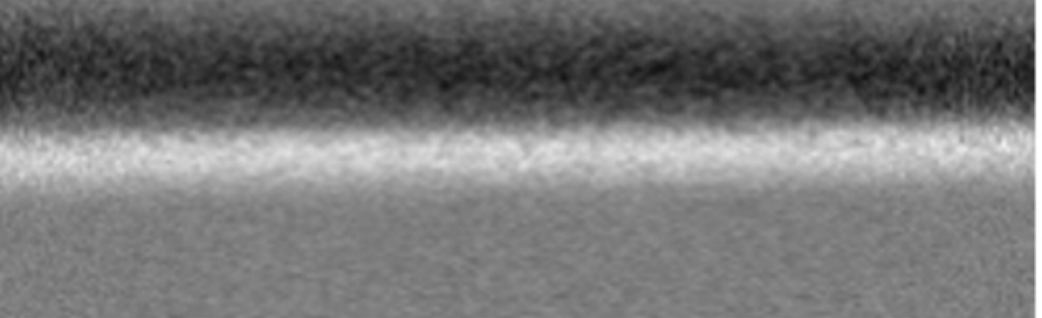

$0^{\circ}$

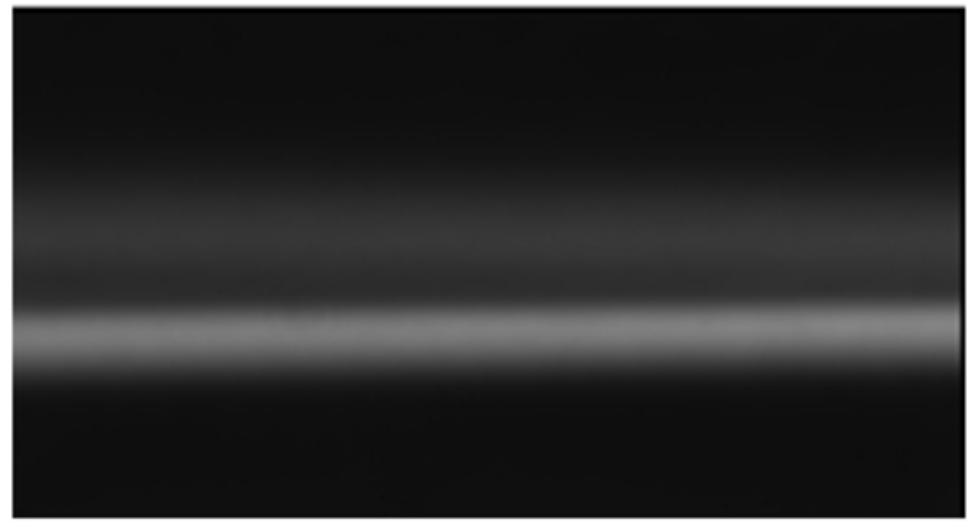

$-4^{0}$

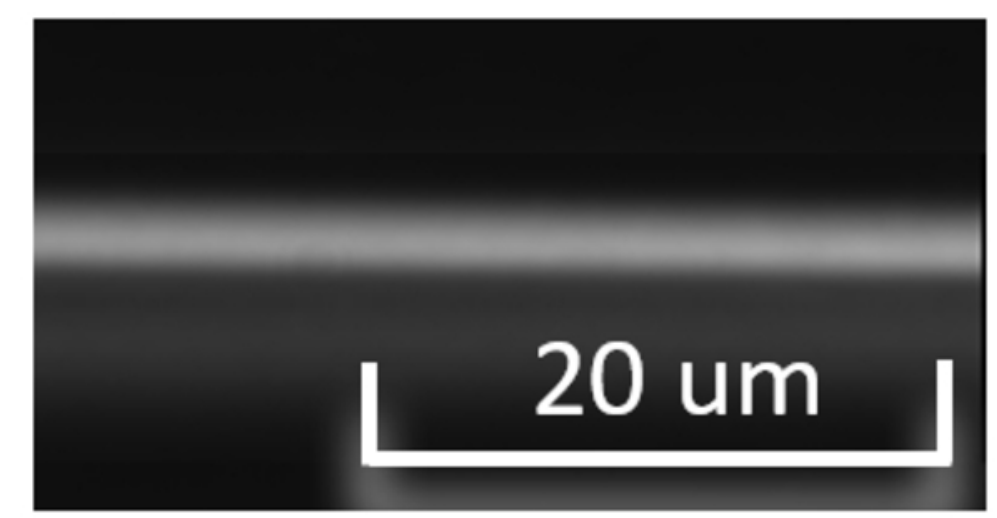




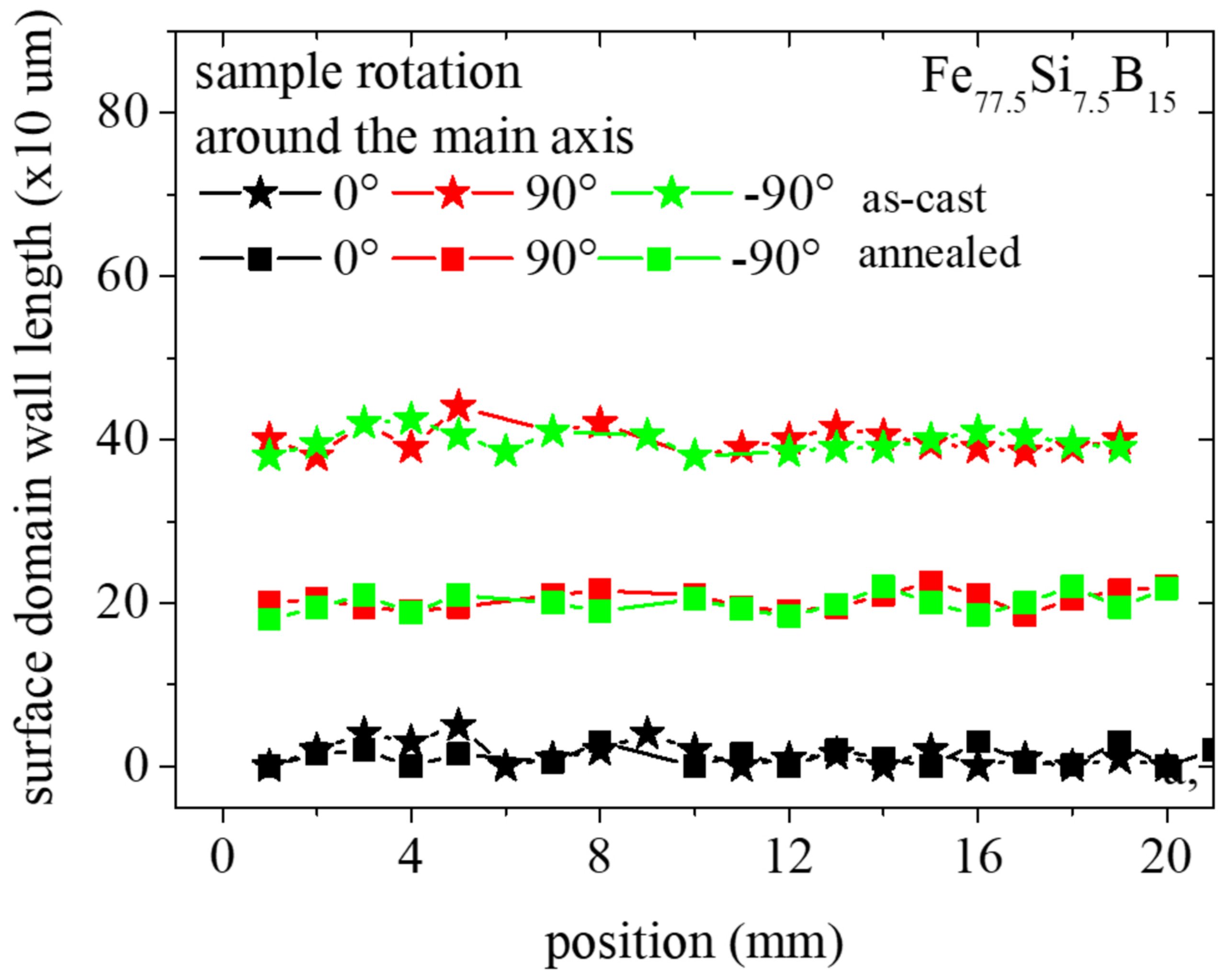



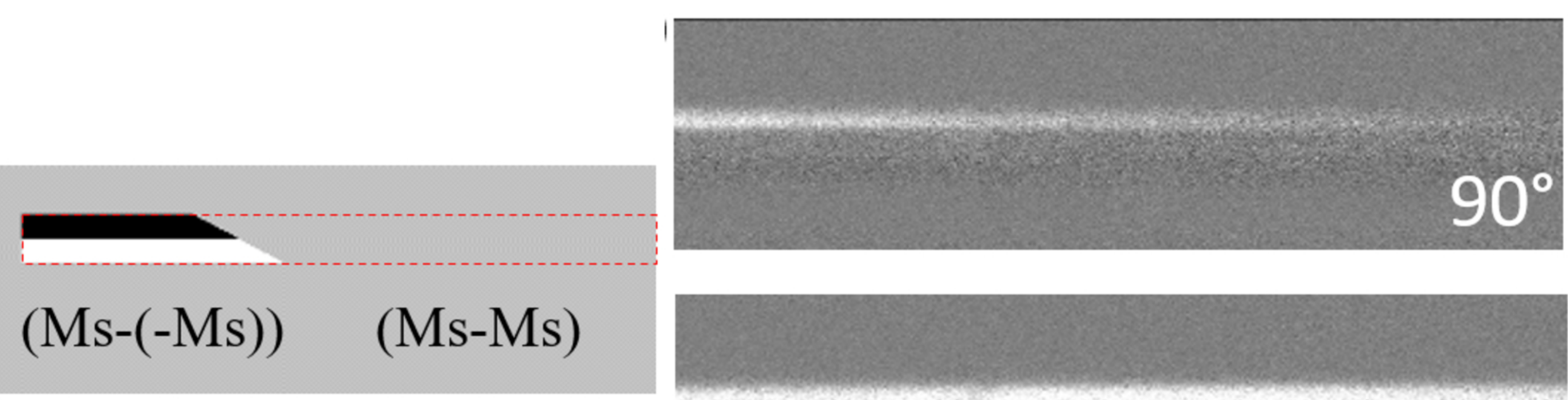

(Ms-(-Ms)) (Ms-Ms)
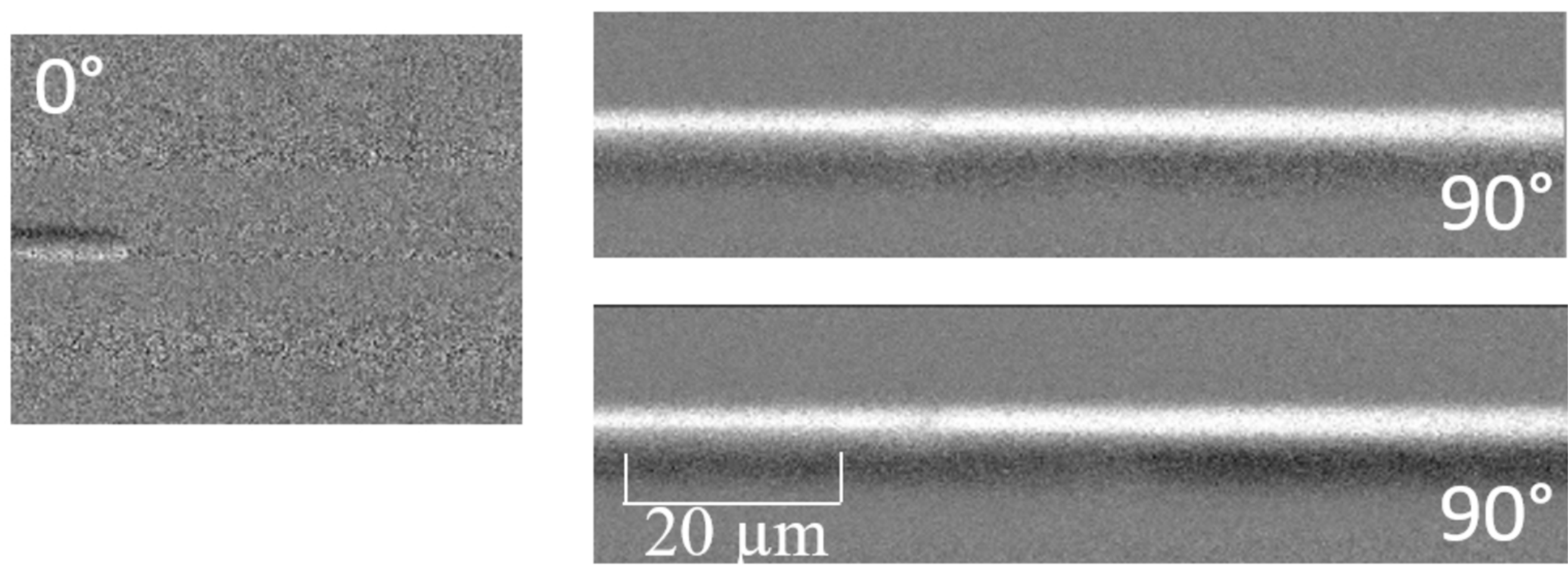


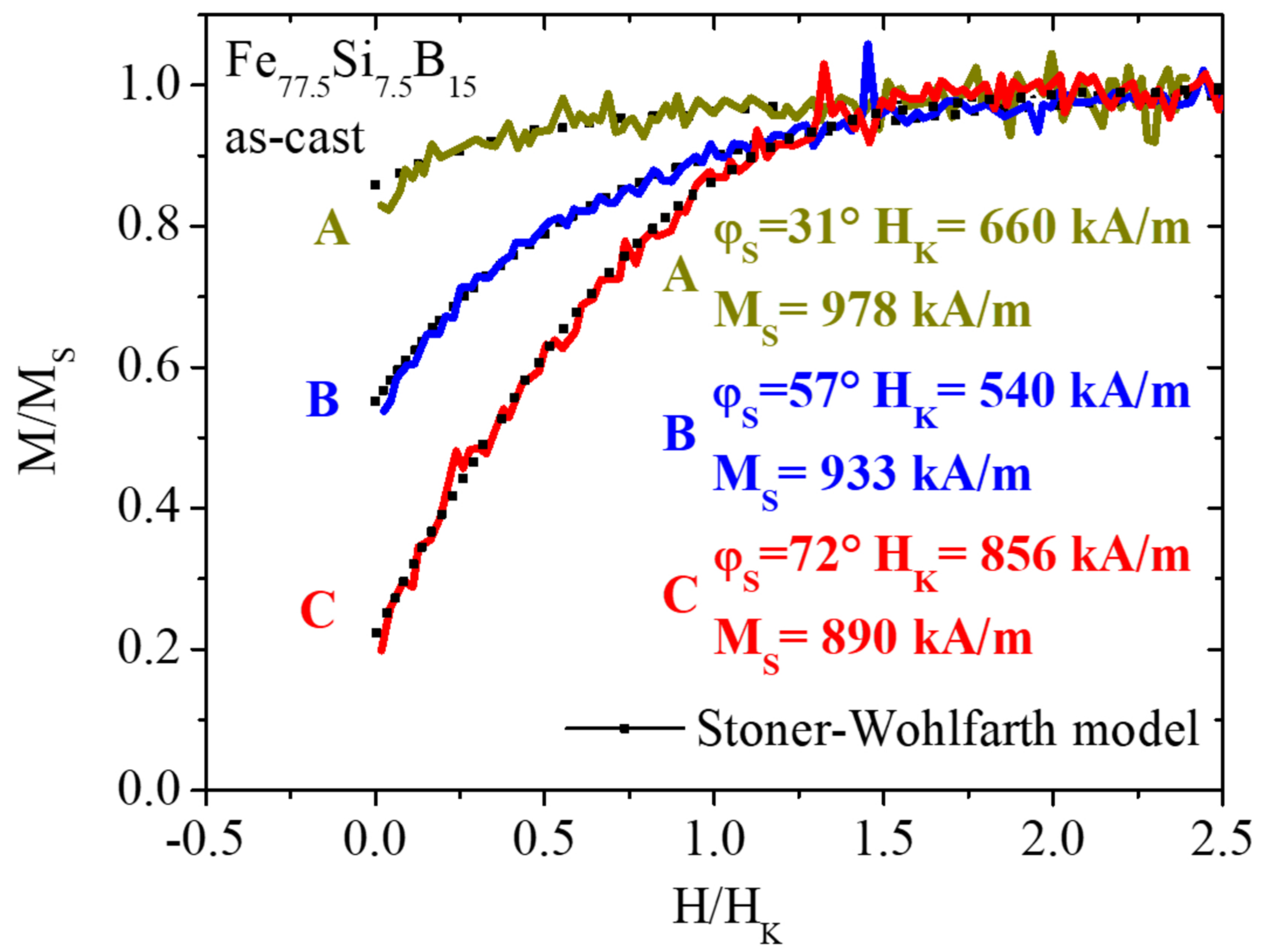




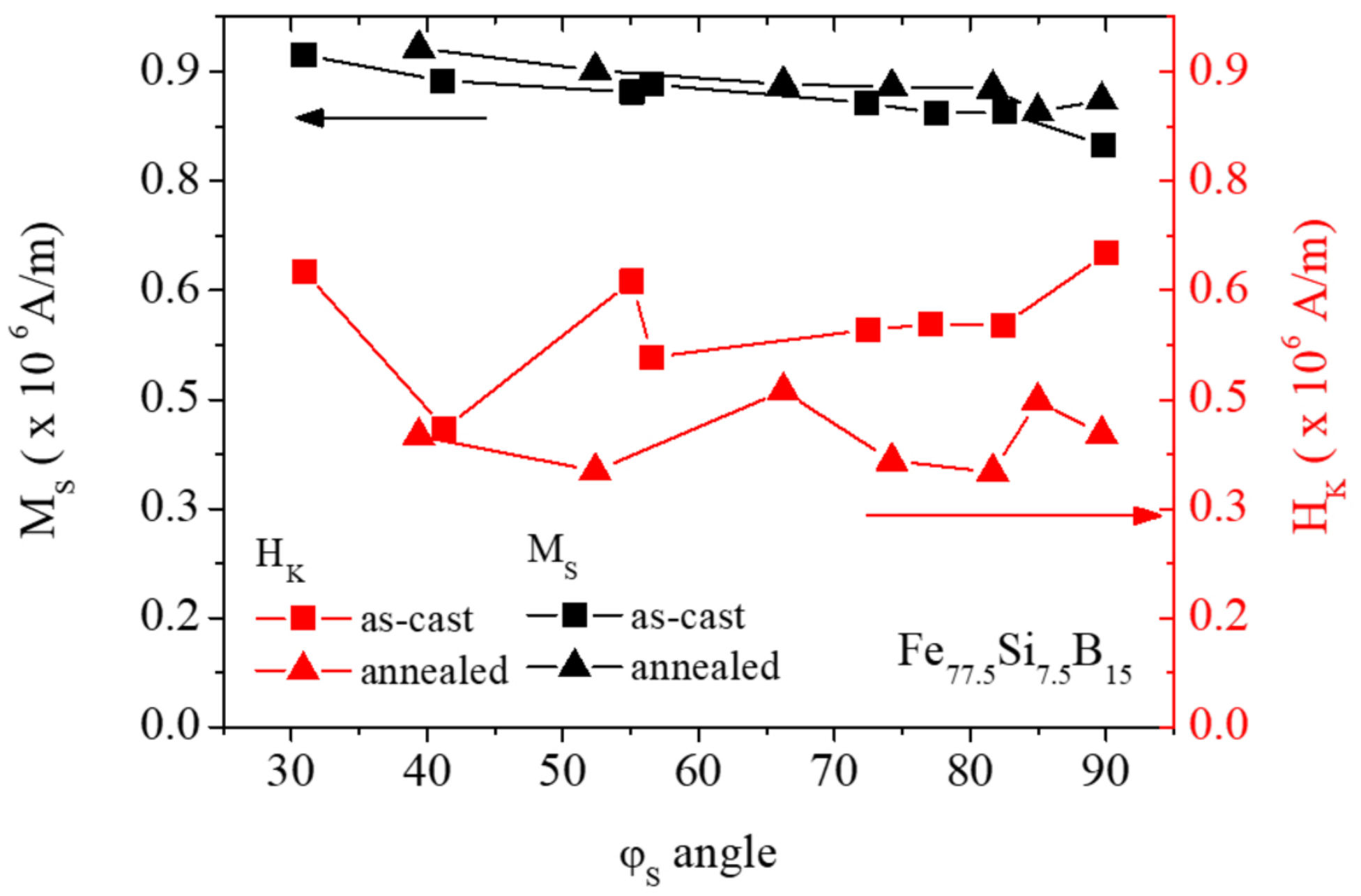




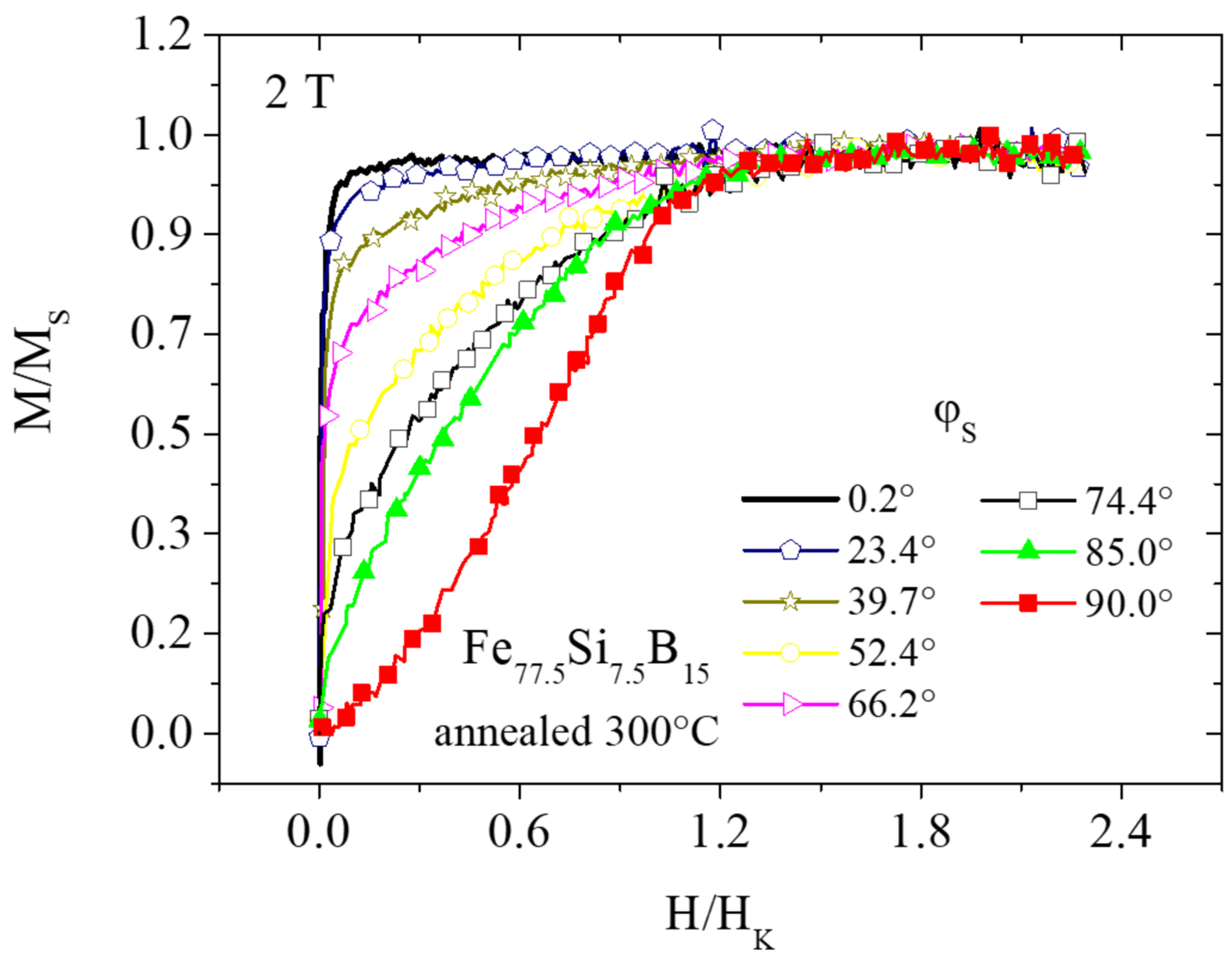




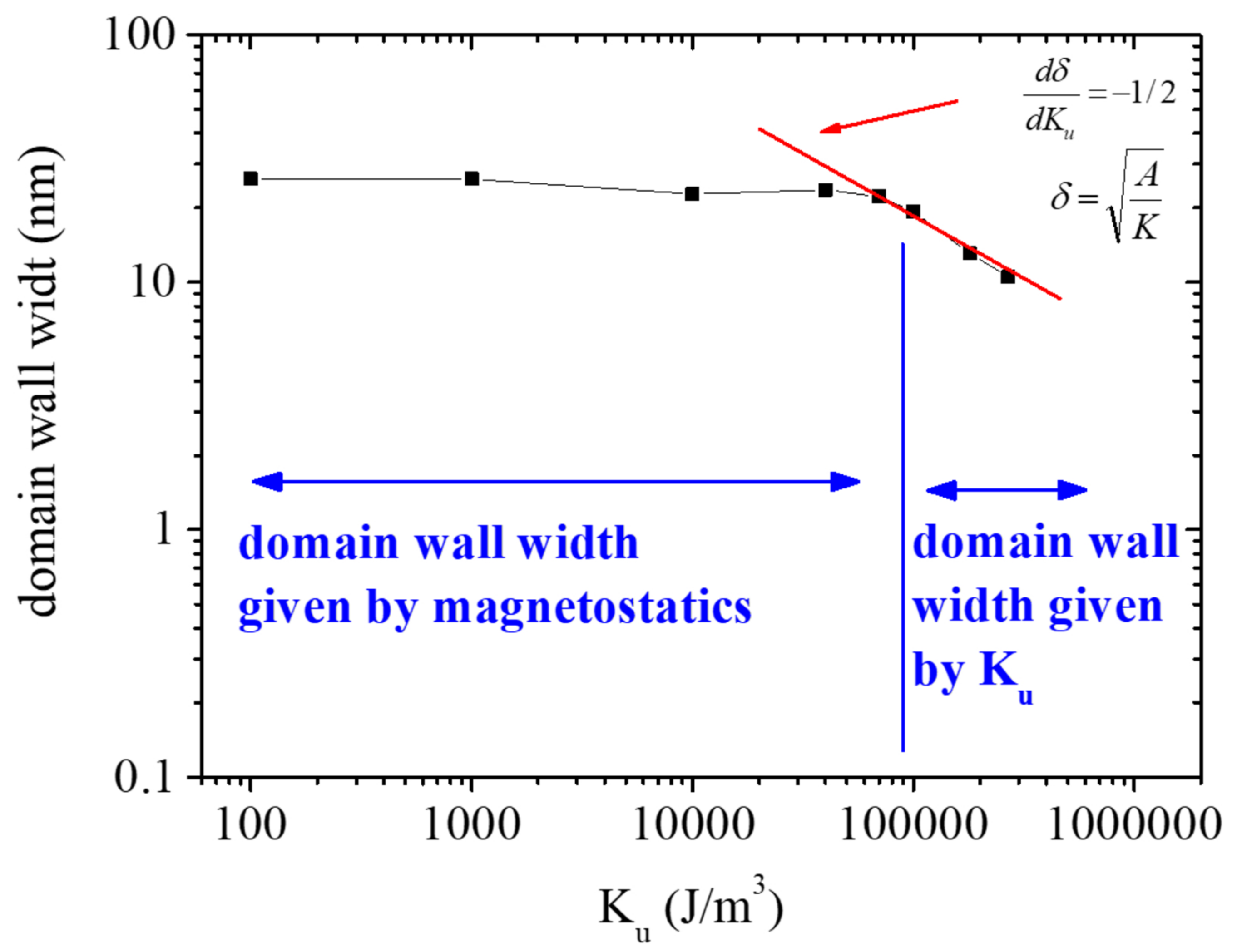




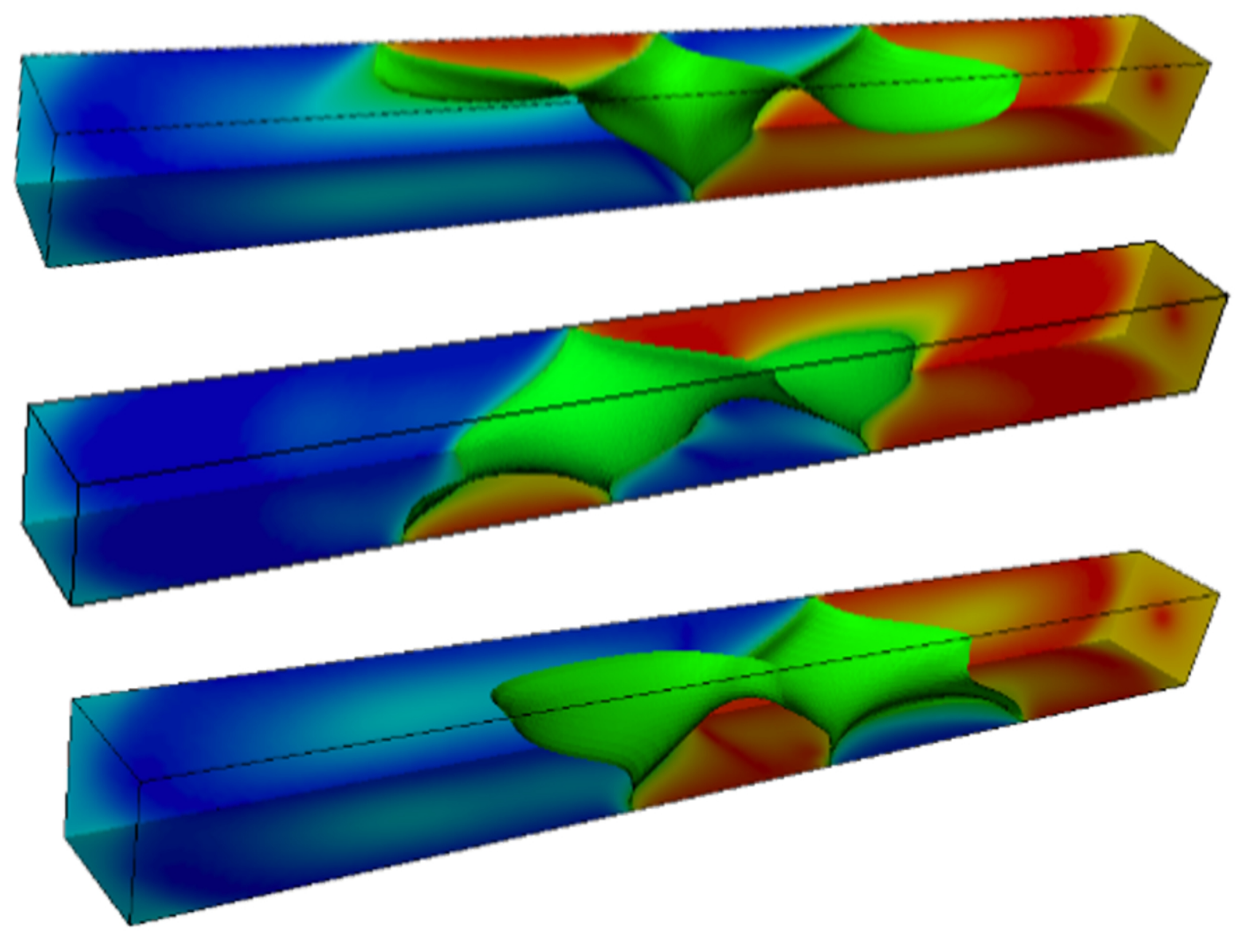




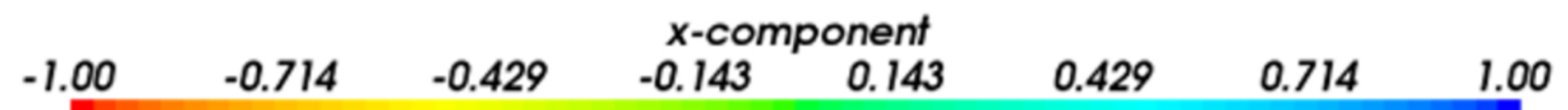

